\title{
A Family of Higher Order Mixed Finite Element Methods for Plane Elasticity
}

\author{
Douglas N. Arnold ${ }^{\star}$, Jim Douglas, Jr. ${ }^{2}$, and Chaitan P. Gupta ${ }^{3}$ \\ ${ }^{1}$ Department of Mathematics, University of Maryland, College Park. MD 20742, USA \\ 2 Department of Mathematics, University of Chicago, Chicago, IL 60637, USA \\ ${ }^{3}$ Department of Mathematics, Northern Illinois University, DeKalb, IL 6011S, USA
}

\begin{abstract}
Summary. The Dirichlet problem for the equations of plane elasticity is approximated by a mixed finite element method using a new family of composite finite elements having properties analogous to those possessed by the Raviart-Thomas mixed finite elements for a scalar, second-order elliptic equation. Estimates of optimal order and minimal regularity are derived for the errors in the displacement vector and the stress tensor in $L^{2}(\Omega)$, and optimal order negative norm estimates are obtained in $H^{s}(\Omega)^{\prime}$ for a range of $s$ depending on the index of the finite element space. An optimal order estimate in $L^{\infty}(\Omega)$ for the displacement error is given. Also, a quasioptimal estimate is derived in an appropriate space. All estimates are valid uniformly with respect to the compressibility and apply in the incompressible case. The formulation of the elements is presented in detail.
\end{abstract}

Subject Classifications: AMS(MOS): 65N30, CR: G1.8.

\section{Introduction}

An isotropic, homogeneous, linearly elastic material in a state of plane strain can be described by the system of partial differential equations

$$
\begin{aligned}
\underset{\approx}{\sigma}=2 \mu \underset{\approx}{g}(u)+\lambda \operatorname{tr}[\underset{\approx}{\varepsilon}(u)] \underset{\approx}{\operatorname{div}} \underset{\sim}{\sigma}=\underset{\sim}{f} & \text { in } \Omega, \\
& \text { in } \Omega,
\end{aligned}
$$

where $\Omega$ is a bounded domain in $\mathbb{R}^{2}, \underline{u}: \Omega \rightarrow \mathbb{R}^{2}$ is the displacement vector, $g: \Omega \rightarrow \mathbb{R}^{2 \times 2}$ is the stress tensor, and $\xi(u)$ is the strain tensor given by $\varepsilon_{i j}(u)$ $=\frac{1}{2}\left(\partial u_{i} / \partial x_{j}+\partial u_{j} / \partial x_{i}\right)$. Both tensors are symmetric. The vector $f$ denotes the forces applied to the body and the real numbers $\mu>0$ and $\lambda \geqq 0$ (the possibility that $\lambda=+\infty$, corresponding to an incompressible material, will also be consid-

* This work was performed while Professor Arnold was a NATO Postdoctoral Fellow 
ered below) are the Lamé constants specifying the material properties. The trace operator applied to a tensor $\tau$ is given by

$$
\operatorname{tr}(\tau)=\tau_{11}+\tau_{22}=\underset{\approx}{\tau}: \underset{\approx}{\delta}
$$

where $\underset{\approx}{\delta}=\left(\delta_{i j}\right)$ is the $2 \times 2$ identity matrix, and the colon denotes the scalar product

$$
\underset{\approx}{\xi}: \underset{\approx}{\tau}=\sum_{i, j=1}^{2} \xi_{i j} \tau_{i j}
$$

Finally, div denotes the differential operator

$$
\operatorname{div} \underset{\sim}{\sigma}=\left(\frac{\partial \sigma_{11}}{\partial x}+\frac{\partial \sigma_{12}}{\partial y}, \frac{\partial \sigma_{21}}{\partial x}+\frac{\partial \sigma_{22}}{\partial y}\right) .
$$

Below we shall also use the elementary differential operators

$$
\begin{aligned}
& \operatorname{div} \underline{v}=\frac{\partial v_{1}}{\partial x}+\frac{\partial v_{2}}{\partial y}, \\
& \underset{\sim}{\operatorname{grad} \varphi} \varphi=\left(\frac{\partial \varphi}{\partial x}, \frac{\partial \varphi}{\partial y}\right), \\
& \operatorname{curl} \varphi=\left(\frac{\partial \varphi}{\partial y}, \frac{-\partial \varphi}{\partial x}\right), \\
& \operatorname{curl} v=\left(\begin{array}{cc}
\frac{\partial v_{1}}{\partial y} & \frac{-\partial v_{1}}{\partial x} \\
\frac{\partial v_{2}}{\partial y} & \frac{-\partial v_{2}}{\partial x}
\end{array}\right), \\
& \operatorname{airy} \varphi=\operatorname{curl}_{\approx} \operatorname{curl} \varphi \text {, } \\
& \Delta \varphi=\operatorname{div} \underset{\sim}{\operatorname{grad} \phi}, \\
& \underline{\Delta v}=\left(\Delta v_{1}, \Delta v_{2}\right) \text {. }
\end{aligned}
$$

For simplicity we shall restrict ourselves to the homogeneous Dirichlet problem. That is, we supplement $(1.1 \mathrm{a}, \mathrm{b})$ by

$$
\underline{u}=\mathbf{0} \quad \text { on } \partial \Omega \text {. }
$$

The boundary $\partial \Omega$ is assumed to be smooth. The precise requirements can be easily obtained by close inspection of the arguments below.

For any space $X$ set $\underset{\sim}{X}=X \times X, \underset{\approx}{X}=\left\{\underset{\approx}{\tau}=\left(\tau_{i j}\right)_{1 \leqq i, j \leqq 2} \in X^{2 \times 2}: \tau_{12}=\tau_{21}\right\}$. FuIther, let

$$
\begin{aligned}
& \underline{V}=\underline{L}^{2}(\Omega)=L^{2}(\Omega) \times L^{2}(\Omega), \\
& \underset{\approx}{H}=\underset{\approx}{H}(\operatorname{div} ; \Omega)=\left\{\tau \in \underset{\approx}{L^{2}}(\Omega): \operatorname{div} \underset{\sim}{\tau \in V}\right\} .
\end{aligned}
$$

The norm on $\underset{\approx}{H}$ is given by $\|g\|_{W}^{2}=\|g\|_{0}^{2}+\|\operatorname{div} g\|_{0}^{2}$. Set

$$
a(\xi, \tau)=\int_{\Omega}\left[\frac{1}{2 \mu} \xi: \tau-\frac{\lambda}{4 \mu(\mu+\lambda)} \operatorname{tr}(\xi) \operatorname{tr}(\tau)\right] d x
$$


To derive a weak form of the Dirichlet problem (1.1) we solve (1.1 a) for $\varepsilon(u)$ and test the resulting equation against $\underset{\approx}{\tau} \in \underset{\approx}{H}$ and integrate the term $\underset{\approx}{\varepsilon(u)}: \underset{\tau}{\tau}$ by

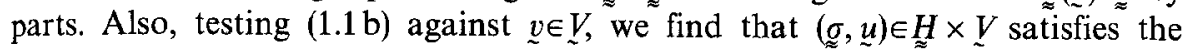
system

$$
\begin{aligned}
& a(\underset{\sim}{\sigma}, \underset{\approx}{\tau})+(\operatorname{div} \underset{\approx}{\tau}, \underline{u})=0 \quad \underset{\approx}{\tau} \in \underset{\approx}{H}, \\
& (\operatorname{div} g, v)=(f, v), \quad \underline{\sim} \in \underset{\sim}{v} .
\end{aligned}
$$

It is this formulation of the Dirichlet problem that we shall consider. Notice that (1.2) makes sense for $\mu \in(0, \infty)$ and $\lambda \in[0, \infty]$. The case $\lambda=\infty$, which corresponds to an incompressible material, requires a further condition, which will be discussed below in Sect. 2 .

The object of this paper is to approximate the solution of (1.2) by a mixed finite element procedure. It is well known [2] that subspaces $\underset{\approx}{H} \times V_{h}$ of $\underset{\approx}{H} \times V$ must be chosen carefully in order that the analogous finite-dimensional saddle point problem, i.e., to find $\left\{g_{h}, \underline{u}_{h}\right\} \in \underset{\sim}{H_{h}} \times V_{h}$ such that

$$
\begin{array}{rlrl}
a\left(g_{h}, \underset{\tau}{\tau}\right)+\left(\operatorname{div} \underset{\approx}{\tau}, u_{h}\right) & =0, & & \underset{\approx}{\tau} \in \underset{\approx}{H}, \\
\left(\operatorname{div} \underset{\approx}{g_{h}}, v\right) & =(f, \underline{v}), & \underline{v} \in V_{h},
\end{array}
$$

is not only solvable but has as solution a good approximation to the solution of (1.2). Johnson and Mercier [8] have introduced two composite, first-degree stress elements, one triangular and the other quadrilateral, that satisfy the Brezzi conditions when combined with a linear or bilinear element for the displacement. For each of their elements, there exists a projection $\Pi_{h}: \underset{\approx}{H^{1}}(\Omega) \rightarrow \underset{\approx}{H}$ having the property that

$$
\left(\operatorname{div}\left(\underset{\sim}{\tau}-\Pi_{h} \tau\right), v\right)=0, \quad \underline{v} \in V_{h} .
$$

Raviart and Thomas [10] also constructed a projection $\Pi_{h}$ for their mixed elements for scalar, second order elliptic problems satisfying the analogue of (1.4). In addition their elements possess a second important property, the analogue of which is shared by our elements as well. Namely, we shall have

$$
\operatorname{div} \underset{\approx}{H_{h}} \subset V_{h}
$$

and hence, letting $P_{h}: V \rightarrow V_{h}$ denote the $\stackrel{\sim}{2}^{2}(\Omega)$-projection,

$$
\left(\operatorname{div} \underset{\approx}{\tau}, \underset{v}{v}-P_{h} \underline{v}\right)=0, \quad \underset{\approx}{\tau} \in \underset{\approx}{H} \text {. }
$$

A direct consequence of (1.4) and (1.5) is that the projections $I_{h}$ and $P_{h}$ are related by the equation

$$
\operatorname{div} \Pi_{h}=P_{h} \operatorname{div}: \underset{\sim}{H}{ }^{1}(\Omega) \rightarrow V_{h} ;
$$

i.e., the following diagram commutes: 


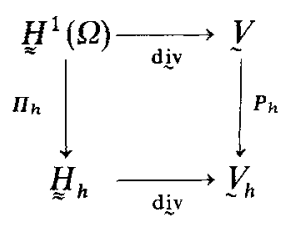

Note that, since $\operatorname{div}_{\widetilde{\sim}}^{\underset{\sim}{H}}{ }^{1}(\Omega)=\underset{V}{V}$ and $P_{h} V=V_{h}$ it follows that $\operatorname{div}_{\sim} \underset{\approx}{H}=V_{h}$; consequently, $\operatorname{div} \Pi_{h}=P_{h} \operatorname{div}$ is onto $V_{h}$.

The properties analogous to (1.4)-(1.7) for the Raviart-Thomas spaces, in combination with their approximation properties, allowed a simple convergence analysis $[10,15]$ and were further exploited by Falk and Osborn [7] to give $L^{2}$ estimates for each variable and by Douglas and Roberts to demonstrate both global $[4,5]$ and interior estimates in $L^{2}(\Omega)$ and $H^{-s}(\Omega)$, as well as superconvergence estimates. The Johnson-Mercier elements [8] do not satisfy (1.5)-(1.7), so much of that program is not feasible.

We shall introduce a family of elements satisfying (1.4) and (1.5) (and hence also (1.6) and (1.7)). The spaces will be associated with an index $k \geqq 2$ (the degree of the piecewise polynomial stress approximants - the displacements will be approximated by piecewise polynomials of degree $k-1$ ) and a triangulation $\mathscr{T}_{h}$ of $\Omega$. Let $\theta$ be a fixed positive number. For each of a sequence of positive numbers $h$ clustering at zero let $\mathscr{T}_{b}$ be a triangulation satisfying

(1.8a) each $K \in \mathscr{T}_{h}$ is either a triangle or else a curvilinear triangle with one curved edge lying along $\partial \Omega$;

(1.8b) $\bigcup_{K \in \mathscr{T}_{h}} K=\bar{\Omega}$

(1.8c) two distinct intersecting elements of $\mathscr{T}_{h}$ meet in a common edge or vertex;

(1.8d) each $K \in \mathscr{T}_{h}$ is star-shaped with respect to each point in the disc of diameter $\theta \operatorname{diam}(K)$ centered at the centroid of the three vertices of $K$;

(1.8e) $\operatorname{diam}(K) \leqq h, K \in \mathscr{T}_{h}$.

(Note that, unmodified, the word triangle refers to a closed nondegenerate straight-edged triangle, i.e., the closed convex hull of three noncollinear points.) Property $(1.8 \mathrm{~d})$ is a regularity hypothesis and reduces to the familiar minimal angle condition for triangles with three straight edges. Note that we do not assume quasiuniformity. We shall construct spaces

$$
\underset{\approx}{H} \times \underline{V}_{h}=\underset{\approx}{H_{h}}\left(k, \mathscr{T}_{h}\right) \times \underline{V}_{h}\left(k, \mathscr{T}_{h}\right) \subset \underset{\approx}{H} \times \underline{V}
$$

which satisfy (1.4) and (1.5) and also the following approximation properties $\left(\|\cdot\|_{s}\right.$ denotes the norm in $\left.H^{s}(\Omega)^{n}\right)$ :

$$
\begin{aligned}
\left\|g-\Pi_{h} g\right\|_{0} \leqq Q\|q\|_{r} h^{r}, & 1 \leqq r \leqq k+1, \\
\left\|\operatorname{div}\left(\underset{\sigma}{\sigma}-\Pi_{h} g\right)\right\|_{-s} \leqq Q\|\operatorname{div} g\|_{r} h^{r+s}, & 0 \leqq r, s \leqq k \\
\left\|\underline{u}-P_{h} \underline{u}\right\|_{-s} \leqq Q\|u\|_{r} h^{r+s}, & 0 \leqq r, s \leqq k .
\end{aligned}
$$


Note that $(1.9 \mathrm{~b})$ is redundant, as it follows from $(1.9 \mathrm{c})$ and (1.7). The space $V_{h}$ will consist of piecewise polynomial vectors of degree $k-1$, without continuity constraints across the edges of the triangles $K \in \mathscr{T}_{h}$. In order to form the space $\underset{\approx}{H_{h}}$ first subdivide each triangle $K \in \mathscr{T}_{h}$ by adding the centroid of its vertices as vertex, and consider the collection of composite triangles that result. Let us consider one such composite, say,

Then,

$$
K=T_{1} \cup T_{2} \cup T_{3}
$$

$$
\left.\underset{\approx}{H_{h}}\right|_{T_{\mathbf{i}}}=\underset{\approx k}{P_{k}}\left(T_{i}\right)
$$

(Here and in the sequel $P_{k}$ is used to denote the space of polynomial functions on $\mathbb{R}^{2}$ of degree at most $k$. For any set $S \subset \mathbb{R}^{2}, P_{k}(S)$ is the space of their restrictions to $S$.) Moreover, any element in $\left.\underset{\approx}{H}\right|_{K}$ must have continuous normal components across the three internal edges, and we shall show that we can require (1.5); i.e., the divergence of an element in $\underset{\approx}{H_{h}}$ is a polynomial vector on each $K$, not just on the microtriangles. The degrees of freedom for $\underset{\approx}{\xi \in H_{h}}$ on $K$ will be the following:

$$
\begin{aligned}
& \int_{e}(\underset{\approx}{\xi} n) \cdot \varphi d s, \quad \underline{\varphi \in P_{k}}(e), \quad \text { for each edge } e \text { of } \partial T,
\end{aligned}
$$

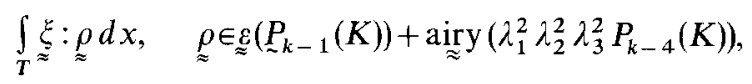

where the $\lambda_{i}$ are the barycentric coordinate functions of $K$. We shall show that we can select $\left.\underset{\approx}{H}\right|_{T}$ such that

$$
\begin{gathered}
\left.\underset{\approx}{H_{h}}\right|_{K} \supset \underset{\approx}{\underset{P}{P}}(K), \\
\operatorname{dim}\left(\left.\underset{\approx}{H_{h}}\right|_{K}\right)=\operatorname{dim}(\underset{\approx}{P}(K))+3=\frac{3}{2} k^{2}+\frac{9}{2} k+6 .
\end{gathered}
$$

Note that $\left.\underset{\approx}{H_{h}}\right|_{K}$ has dimension exactly three greater than the minimum required for the approximation property (1.9a) to hold, whatever the choice of $k$. For $k=2, \operatorname{dim}\left(\left.{\underset{z}{H}}_{h}\right|_{K}\right)=21$, and the degrees of freedom are the eighteen arising from the requirement (1.10a) that $\underset{\approx}{H} \subset \underset{\approx}{H}$ (cf. [8]) and the moments of the three functions $\xi_{11}, \xi_{12}=\xi_{21}$, and $\xi_{22}$ over $K$. For computational purposes point values could be used in place of moments.

We shall prove the following error bounds:

$$
\left\|\underset{\sim}{u}-\underline{u}_{h}\right\|_{-s} \leqq \begin{cases}Q\|\underline{u}\|_{r} h^{r+s} & \text { for } 2 \leqq r \leqq k \text { and } 0 \leqq s \leqq k-2, \\ Q\|\underline{u}\|_{r+1} h^{r+k-1} & \text { for } 1 \leqq r \leqq k \text { and } s=k-1, \\ Q\|\underline{u}\|_{r+2} h^{r+k} & \text { for } 0 \leqq r \leqq k \text { and } s=k,\end{cases}
$$

$$
\begin{aligned}
& \left\|Q-g_{h}\right\|_{-s} \leqq Q\|\underline{\sim}\|_{r+1} h^{r+s} \quad \text { for } \quad 1 \leqq r \leqq k+1 \text { and } 0 \leqq s \leqq k-1 \text {, } \\
& \left\|\operatorname{div}\left(g-g_{\sigma}\right)\right\|_{-s} \leqq Q\|\operatorname{div} \underset{\sim}{g}\|_{r} h^{r+s} \quad \text { for } \quad 0 \leqq r, s \leqq k .
\end{aligned}
$$

The constant $Q$ in these estimates is independent of the compressibility of the material. This is a distinct advantage of the method. Specifically, let $0<\mu_{0}<\mu_{1}<\infty$ and assume that the first Lamé constant $\mu \in\left[\mu_{0}, \mu_{1}\right]$. The letter 
$Q$ will denote a generic constant which may depend only on $\Omega, \mu_{0}, \mu_{1}, \theta$, and $k$; but, in particular, it is independent of $h$ and $\lambda$.

Note that each $L^{2}(\Omega)$ estimate is optimal, both with respect to the rate of convergence and to the regularity demanded of the solution. In fact, the same is true for the negative norm estimates for $g-\sigma_{n}$ and its divergence. For the displacement, additional regularity is required in order to obtain the maximal rate of convergence. Note that the rate of convergence for $\sigma-g_{h}$ is limited to $O\left(h^{2 k}\right)$, instead of $O\left(h^{2 k+2}\right)$ that occurs for the difference of $\sigma$ and its $L^{2}$ projection into ${ }_{z}$. This limitation reflects the fact that the displacement and the divergence of the stress are being approximated by piecewise polynomials of degree $k-1$. In contrast, Douglas and Roberts [5] were able to obtain $O\left(h^{2 k+2}\right)$ rates for both the original dependent variable and its associated velocity field when the Raviart-Thomas space of index $k$ is employed for the scalar elliptic problem; however, it should be remembered that the space for the vector field includes some polynomials of degree $k+1$ and the scalar part contains all polynomials of degree $k$.

In addition to the $L^{2}$-based estimates, we shall show under the further restriction of the triangulation $\mathscr{T}_{h}$ that, for some $\rho>0$,

$$
\rho h \leqq \operatorname{diam}(K) \leqq h, \quad K \in \mathscr{T}_{h},
$$

it follows that

$$
\left\|\underline{u}-\underline{u}_{h}\right\|_{0, \infty} \leqq Q\left\{\|\underline{u}\|_{r, \infty}+\|\underline{u}\|_{r+1}\right\} h^{r} \quad \text { for } 1 \leqq r \leqq k+1 .
$$

This $L^{\infty}$-estimate shows that the maximal rate of convergence occurs if the solution is slightly more regular than required for approximation alone. A corresponding estimate has been found by Scholz [11] and Douglas-Roberts [5] for the scalar variable in the second order elliptic problem. Scholz [12,13] has recently obtained an estimate not requiring the additional regularity for the Dirichlet problem.

The organization of the remainder of the paper is as follows. In the next section we establish regularity for the continuous problem uniformly in $\lambda$ and comment on the incompressible case. Then, assuming (1.4)-(1.9), we establish the $L^{2}$ and $H^{-s}$ estimates stated in (1.12). Then the $L^{\infty}$ estimate (1.14) is obtained as a trivial consequence of the duality argument employed to bound $\tilde{u}-u_{h}$. In the remainder of the paper we construct the spaces $H_{k}$ and $\underline{Z}_{h}$ and the projection operator $\Pi_{h}$ and verify the required properties (1.4), (1.5), (1.9a), and $(1.9 \mathrm{c})$. Finally, we indicate a composite quadrilateral element; this is done more for completeness than for actual applicability.

The authors would like to thank Professor Franco Brezzi for a number of helpful suggestions related to this research.

\section{The Differential Problem}

If ( $1.1 \mathrm{a})$ is substituted into $(1.1 \mathrm{~b})$, we recover the Lamé equation

$$
\mu \underline{\Delta} \underset{u}{u}+(\lambda+\mu) \operatorname{grad} \operatorname{div} \underline{u}=f .
$$


Together with the homogeneous Dirichlet boundary condition $(1.1 \mathrm{c})$ this is a strictly coercive, strongly elliptic boundary value problem. Hence well-known regularity theory applies. Less obvious is that the regularity estimates hold uniformly for $\lambda \in[0, \infty)$. We prove this result here. An analogous result for the Neumann problem is proved in [16].

Thêrem 2.1. Let $\mu_{0}<\mu_{1}$ be real numbers, and let $s \geqq-1$ be an integer. Suppose that $\mu \in\left[\mu_{0}, \mu_{1}\right], \lambda \in[0, \infty)$, and $f \in \tilde{H}^{s}(\Omega)$ (where here $\left.\stackrel{H}{H}^{-1}(\Omega)=\mathscr{H}^{1}(\Omega)^{\prime}\right)$. Then there exists a unique solution $(g, u) \in \underset{\approx}{H} \times \underline{V}$ to (1.2). Moreover, $\sigma \in H_{\approx}^{H^{+1}}(\Omega)$ and $\underline{u} \in H^{s+2}(\Omega) \cap \stackrel{H}{1}^{1}$, and there exists a constant $Q$ depending only on $\Omega, \mu_{0}, \mu_{1}$, and s such that

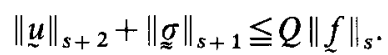

Proof. The standard theory of elliptic partial differential equations ensures the existence and uniqueness of the solution and furnishes the estimate (2.2) uniformly for $\mu \in\left[\mu_{0}, \mu_{1}\right]$ and $\lambda$ in any compact subinterval of $[0, \infty)$. We complete the proof by showing that a constant $Q$ can be found so that (2.2) holds for $\lambda$ in a certain unbounded interval of $[0, \infty)$. We shall rely on the regularity theory for the generalized Stokes problem. According to this theory [14, Prop. 2.2], there exists $Q_{0} \in \mathbb{R}$ such that, if $\widehat{L^{2}}(\Omega)=\left\{p \in L^{2}(\Omega) \mid \int_{\Omega} p d x=0\right\}$ and if $(u, p) \in \stackrel{\leftrightarrow}{H}^{1}(\Omega) \times \widehat{L^{2}}(\Omega)$ solves the Stokes equations

$$
\begin{aligned}
\mu \underline{\Delta} \underline{u}+\operatorname{grad} p=f & \text { in } \Omega, \\
\operatorname{div} \underline{u}=g & \text { in } \Omega,
\end{aligned}
$$

for some $f, g \in \sim_{\sim}^{H}(\Omega) \times H^{s+1}(\Omega)$, then $(u, p) \in \underset{\sim}{H^{s+2}}(\Omega) \times H^{s+1}(\Omega)$ and

$$
\|u\|_{s+2}+\|p\|_{s+1} \leqq Q_{0}\left(\|f\|_{s}+\|g\|_{s+1}\right) .
$$

The constant $Q_{0}$ depends only on $\Omega, \mu_{0}, \mu_{1}$, and $s$. Now, suppose $\lambda \geqq 2 Q_{0}-\mu_{0}$ and set

$$
p=(\lambda+\mu) \operatorname{div} \underline{u} .
$$

Then from (2.1) and (2.5) we have (2.3) with $g=(\lambda+\mu)^{-1} p$. Hence, (2.4) yields the bound

$$
\begin{aligned}
& \|\underline{u}\|_{s+2}+\frac{\lambda+\mu}{2}\|\operatorname{div} \underline{u}\|_{s+1} \leqq Q_{0}\left(\|f\|_{s}+\frac{1}{\lambda+\mu}\|p\|_{s+1}\right)-\frac{1}{2}\|p\|_{s+1} \\
& \leqq Q_{0}\|f\|_{s},
\end{aligned}
$$

as $Q_{0} /(\lambda+\mu) \leqq 1 / 2$. Since $g=2 \mu \varepsilon(u)+\lambda(\operatorname{div} \underset{z}{u}) \delta$, this completes the proof of (2.2).

We also remark on the incompressible case, $\lambda=+\infty$. The weak formulation (1.2) remains valid in this case, the form $a(\xi, t)$ now being given by

$$
a(\underset{\approx}{\xi}, \tau)=\int_{\Omega}\left[\frac{1}{2 \mu} \xi: \tau-\frac{1}{4 \mu} \operatorname{tr}(\underset{\approx}{\xi}) \operatorname{tr}(\tau)\right] d x .
$$


In this case, however, $g$ is not uniquely determined by the equations $(1.2 \mathrm{a}, \mathrm{b})$ : we can add any constant multiple of $\delta$ to $g$. We therefore impose the condition

$$
\int_{\Omega} \operatorname{tr}(\sigma) d x=0
$$

Note that, for $\lambda<+\infty,(1.2 \mathrm{c})$ follows from (1.2a) with the choice $\underset{\sim}{\tau}=\underset{\approx}{\delta}$. Hence, in the compressible case this additional condition does not alter the problem (1.2). When $\lambda=+\infty$, it is easily checked that, if the pair $(u, p) \in \dot{H}^{1}(\Omega) \times \widehat{L^{2}}(\Omega)$ solves the Stokes equations (2.3) with $g=0$, then the pair $(2 \mu \underline{g}(u)+p \underset{\sim}{\delta}, \underline{u}) \in \underset{z}{H} \times V$ solves (1.2). And, conversely, if $(g, \underset{\approx}{u}) \in \underset{\approx}{H} \times V$ solves (1.2), then $(u, \operatorname{tr}(g) / 2) \in \stackrel{\circ}{\sim}^{1}(\Omega)$ $\times \widehat{L}(\Omega)$ solves $(2.3)$ with $g=0$. Hence, existence, uniqueness, and regularity of (1.2) in the incompressible case follow from the analogous results for the Stokes system. We conclude that Theorem 2.1 is valid for $\lambda \in[0, \infty]$.

In analogy with $(1.2 \mathrm{c})$ it is natural to impose on the discrete solution the condition

$$
\int_{\Omega} \operatorname{tr}\left(\sigma_{h}\right) d x=0 .
$$

As in the continuous case this condition is redundant if $\lambda<+\infty$ : since $\underset{\approx}{H}$ will contain $\underset{\approx}{\delta}$, it follows from $(1.3 \mathrm{a})$.

We now turn to the error analysis of the method given by (1.3) for the problem (1.2).

\section{The Error Analysis}

Let

$$
\begin{array}{ll}
\underset{\approx}{\xi}=\underset{\approx}{\sigma}-g_{h}, & \underset{\approx}{\zeta}=\Pi_{h} g-\sigma_{\mathfrak{z}}, \\
\underline{\eta}=\underline{u}-\underline{u}_{h}, & \underline{\gamma}=P_{h} \underline{u}-\underline{u}_{h} .
\end{array}
$$

Then, subtracting (1.3) from (1.2) gives the error equations

$$
\begin{aligned}
& a\left(\sum_{\approx}^{\xi}, \tau\right)+(\operatorname{div} \underset{\approx}{\tau}, \eta)=0, \quad \underset{\approx}{\tau \in H_{n}}, \\
& (\operatorname{div} \underset{\approx}{\xi}, v)=0, \quad v \in V_{h} .
\end{aligned}
$$

It follows from (1.4) that $\underset{\approx}{\xi}$ can be shifted to $\underset{\approx}{\zeta}$ in (3.1b); then (1.5) implies that

$$
\operatorname{div} \underset{\approx}{\leftrightarrows}=0 \text {. }
$$

Thus,

$$
\operatorname{div} \underset{\approx}{\xi}=\operatorname{div}\left(g-\Pi_{h} g\right)
$$

and $(1.12 \mathrm{c})$ follows from $(1.9 \mathrm{~b})$.

To derive error estimates for the stress we require two lemmas. In them we use the notation $\tau^{D}$ for the deviatoric of a tensor $\underset{\tau}{\tau}$, defined by

$$
\tau^{D}=\tau-\frac{1}{2} \operatorname{tr}(\tau) \underset{\approx}{\delta}
$$


The norm $\|\cdot\|_{-s}$ denotes the norm in $\left[H^{-s}(\Omega)\right]^{n}=\left[H^{s}(\Omega)^{\prime}\right]^{n}$ for $s$ a nonnegative integer.

Lemma 3.1. Let $s$ be a nonnegative integer. Then, there exists a constant $Q$ depending only on $s$ and $\Omega$ such that

$$
\|\underset{\approx}{\tau}\|_{-s} \leqq Q\left[\left\|\tau_{\approx}^{D}\right\|_{-s}+\|\underset{\sim}{\operatorname{div}} \underset{\approx}{\tau}\|_{-s-1}\right]
$$

for all $\underset{\approx}{\tau} \in \underset{\sim}{H}$ such that $\int_{\Omega} \operatorname{tr}(\tau) d x=0$.

Proof. Clearly it suffices to bound $\|\operatorname{tr}(\tau)\|_{-s}$ by the right hand side of (3.3). Now there exists a nonzero function $\varphi \in H^{s}(\Omega)$ such that

$$
\|\operatorname{tr}(\tau)\|_{-s}\|\varphi\|_{s}=\int \operatorname{tr}(\tau) \varphi d x
$$

Since $\int_{\Omega} \operatorname{tr}(\tau) d x=0$, we can assume $\int_{\Omega} \varphi d x=0$. Therefore, there exists $\underline{v \in \dot{H}^{1}}(\Omega) \cap \underline{H}^{s+1}(\Omega)$ such that $\operatorname{div} \underset{v}{v}=\varphi$ and $\|\underline{\underline{v}}\|_{s+1} \leqq Q\|\varphi\|_{s}$ (cf. Lemma 5.4 .2 of $[1]$ and its proof). Thus,

$$
\begin{aligned}
& (2 Q)^{-1}\|\operatorname{tr}(\tau)\|_{-s}\|\underline{v}\|_{s+1} \leqq \frac{1}{2} \int_{\Omega} \operatorname{tr}(\tau) \operatorname{div} \underset{\sim}{v} d x \\
& =\frac{1}{2} \int_{\Omega} \operatorname{tr}(\tau) \oint_{\approx}: \xi(v) d x=\int_{\Omega}\left(\tau-\tau_{\approx}^{D}\right): \xi(v) d x \\
& =-\int_{\Omega}[\underset{\tau}{\tau}: \underset{\approx}{\varepsilon}(v) d x+\underset{\sim}{\operatorname{div}} \underset{\sim}{\tau} \cdot \underset{\sim}{v}] d x \\
& \leqq\left[\|\|_{\tilde{\tau}}^{D}\left\|_{-s}+\right\| \underset{\sim}{\operatorname{div}} \underset{\sim}{\tau} \|_{-s-1}\right]\|v\|_{s+1} .
\end{aligned}
$$

Lemma 3.2. Let $s$ be a nonnegative integer. Then, for $\underset{\approx}{\tau} \underset{\approx}{H}$,

$$
\begin{aligned}
& \left\|\tau_{\approx}^{D}\right\|_{0}^{2} \leqq 2 \mu a(\underset{\approx}{\tau}, \underset{\approx}{\tau}), \\
& \left\|\tau_{\tilde{\tau}}^{D}\right\|_{-s} \leqq 2 \mu \sup _{0 \neq \rho \in H^{s}} \frac{a(\underset{\tau}{\tau}, \rho)}{\left.\|\underset{\approx}{\rho}\|_{s}\right)} .
\end{aligned}
$$

Proof. There exists a $\underset{\approx}{\rho \in H^{s}}(\Omega)$ such that

Since $\operatorname{tr}\left(\rho^{D}\right)=0$,

$$
\left\|\tau^{D}\right\|_{-s}\|\underset{\approx}{\rho}\|_{s}=\int_{\Omega} \underset{\approx}{\tau^{D}}: \underset{\approx}{\rho} d x=\int_{\Omega} \tau_{\approx}^{D}: \underset{\approx}{\rho} d x
$$

moreover,

$$
a\left(\tau, \underset{\approx}{\rho} \rho^{D}\right)=\frac{1}{2 \mu} \int_{\Omega}{\underset{\tau}{\approx}}^{D}:{\underset{\sim}{\rho}}^{D} d x
$$

Thus,

$$
\left\|\rho_{\approx}\right\|_{\|_{s}} \leqq\|\rho\|_{s} \text {. }
$$

$$
\left\|\tau_{\widetilde{D}}^{D}\right\|_{-s} \leqq 2 \mu \frac{a\left(\underset{\sim}{\tau}, \rho^{D}\right)}{\|\underset{\approx}{\rho}\|_{s}},
$$

proving $(3.4 \mathrm{~b})$. The same argument applies to prove (3.4a). Take $\underset{\approx}{\rho}=\tau^{D}$ and note that 


$$
\begin{aligned}
\left\|\tau_{\approx}^{\tau^{D}}\right\|_{0}^{2} & =2 \mu a\left(\tau^{D}, \tau^{D}\right) \\
& =\int_{\Omega}\left(\tau: \tau-\frac{1}{2} \operatorname{tr}(\tau)^{2}\right) d x \\
& \leqq \int_{\Omega}\left(\underset{\tau}{\tau}: \tau-\frac{\lambda}{2(\mu+\lambda)} \operatorname{tr}(\tau)^{2}\right) d x \\
& =2 \mu a(\tau, \tau) .
\end{aligned}
$$

We are now prepared to bound $\underset{\approx}{\xi}=\underset{\approx}{\sigma}-\sigma_{h}$ in $L_{\approx}^{2}(\Omega)$. By $(1.2 \mathrm{c})$ and $(1.3 \mathrm{c})$, $\int_{\Omega} \operatorname{tr}(\underset{\approx}{\xi}) d x=0$, and hence by the lemmas

$$
\|\underset{\approx}{\xi}\|_{0}^{2} \leqq Q\left[a(\underset{\approx}{\xi} \underset{\approx}{\xi})+\|\operatorname{div} \underset{\approx}{\xi}\|_{-1}^{2}\right]
$$

We have

$$
\|\operatorname{div} \underset{\approx}{\xi}\|_{-1} \leqq Q\left\|_{\approx}\right\|_{r} h^{r}, \quad 1 \leqq r \leqq k+1
$$

by $(1.12 \mathrm{c})$, while

$$
a(\underset{\approx}{\xi}, \underset{\approx}{\xi})=a\left(\underset{\approx}{\xi}, g-\Pi_{h} \sigma\right) \leqq Q\left\|{ }_{\approx}\right\|_{0}\|g\|_{r} h^{r}, \quad 1 \leqq r \leqq k+1
$$

by (3.1 a), (3.2) and (1.9a). Combining (3.5)-(3.7) gives the estimate

$$
\left\|g-g_{h}\right\|_{0} \leqq Q\|g\|_{r} h^{r}, \quad 1 \leqq r \leqq k+1
$$

Next, note that (1.6) allows us to replace $\eta$ by $\underline{\gamma}$ in (3.1 a) to obtain the relation

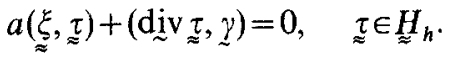

We should like to estimate $\gamma$ in ${\underset{\sim}{H}}^{-s}(\Omega)={\underset{\sim}{H}}^{s}(\Omega)^{\prime}$. Let $0 \leqq s \leqq k$ and let $\psi \in \underset{\sim}{H^{s}}(\Omega)$. Then, there exists $\underset{\approx}{\chi}, \underline{\varphi}) \in \underset{\approx}{H} \times \underline{V}$ such that

$$
\begin{aligned}
& a(\underset{\approx}{\chi}, \tau)+\left(\operatorname{div}_{\approx} \tau, \varphi\right)=0, \quad \underline{\tau} \in \underset{\approx}{\sim}, \\
& \left(\operatorname{div}_{\sim} \underset{\approx}{\chi}, \underline{v}\right)=-(\Psi, v), \quad \underline{v} \in \underline{\sim}, \\
& \int_{\Omega} \operatorname{tr}(\chi) d x=0 \text {. }
\end{aligned}
$$

Moreover, by Theorem 2.1, $\underset{\approx}{\chi \in} \underset{\sim}{H^{s+1}}(\Omega), \underline{\varphi} \in \underset{\sim}{H^{s+1}}(\Omega) \cap \stackrel{\sim}{H}^{1}(\Omega)$, and

$$
\|\chi\|_{s+1}+\|\varphi\|_{s+2} \leqq Q\|\psi\| \|_{s} .
$$

It follows from (1.4) and (3.9) that

Since

$$
\begin{aligned}
& (\gamma, \psi)=-(\underline{\gamma}, \operatorname{div} \underset{z}{\chi})=-\left(\gamma, \operatorname{div} \Pi_{h} \underset{z}{\chi}\right) \\
& =a\left(\xi, \Pi_{h} \chi \underset{\approx}{\chi}\right)=a(\underset{\approx}{\xi}, \underset{\approx}{\chi})+a\left(\underset{\approx}{\xi}, \Pi_{h} \underset{\approx}{\chi}-\underset{\approx}{\chi}\right) .
\end{aligned}
$$

$$
\left.a(\underset{z}{\xi}, \chi)^{\prime}\right)=-(\operatorname{div} \underset{\approx}{\xi}, \varphi)=-\left(\operatorname{div} \underset{\approx}{\xi}, \varphi-P_{h} \varphi\right)
$$

by $(3.10 \mathrm{a})$ and $(3.1 \mathrm{~b})$,

$$
|(\gamma, \psi)| \leqq Q\|\operatorname{div} \underset{\sim}{\xi}\|_{0}\|\varphi\|_{s+2} h^{\min (s+2, k)}+Q\|\xi\|_{0}\|\chi\|_{s+1} h^{s+1},
$$


so that

$$
\|\gamma\|_{-s} \leqq Q\left\{\|\underset{\approx}{\xi}\|_{0} h^{s+1}+\|\operatorname{div} \underset{\approx}{\xi}\|_{0} h^{\min (s+2, k)}\right\}
$$

for $0 \leqq s \leqq k$. Then, it follows from $(1.12 \mathrm{c})$ and (3.8) that

$$
\|y\|_{-s} \leqq Q\|u\|_{r} h^{r+s} \text { for } 2 \leqq r \leqq k+2 \text { and } 0 \leqq s \leqq k-2 .
$$

This inequality can be combined with $(1.9 \mathrm{c})$ to see that

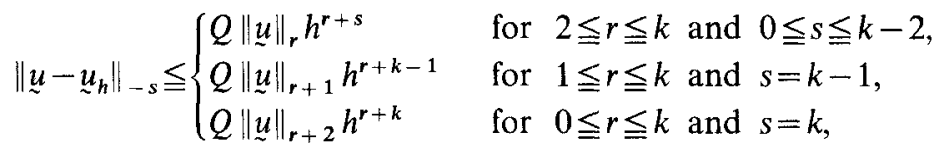

where the estimate for $\|y\|_{-k+2}$ has been used in the last two of the bounds in (3.12). Thus, (1.12a) has been established.

We turn now to obtain the bound (1.12b) for $\underset{\approx}{\sigma}-\sigma_{\boldsymbol{\sigma}}$ in $\underset{\approx}{H^{-s}}(\Omega)$ for $1 \leqq s \leqq k$ -1 . Let $\varphi \in \underset{\approx}{H^{s}}(\Omega)$. Then, by (3.9) and (1.4),

$$
\begin{aligned}
a(\underset{\approx}{\xi}, \underset{\approx}{\varphi}) & =a\left(\underset{\approx}{\xi}, \underset{\approx}{\varphi}-\Pi_{h} \underset{\approx}{\varphi}\right)-\left(\operatorname{div} \Pi_{h} \underset{\approx}{\varphi}, \gamma\right) \\
& =a\left(\underset{\approx}{\xi}, \underset{\approx}{\varphi}-\Pi_{h} \varphi\right)-(\operatorname{div} \underset{\approx}{\varphi}, \underset{\gamma}{\gamma}) \\
& \leqq Q\left\{\|\underset{\approx}{\xi}\|_{0} h^{s}+\|\gamma\|_{-s+1}\right\}\|\underset{\approx}{\varphi}\|_{s},
\end{aligned}
$$

and, by Lemma 3.2,

By (3.8) and (3.11),

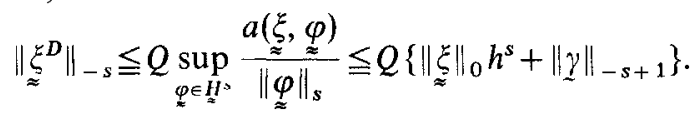

$$
\left\|\xi^{\xi^{D}}\right\|_{-s} \leqq Q\|\underline{u}\|_{r+1} h^{r+s} \quad \text { for } \quad 1 \leqq r \leqq k+1 \quad \text { and } \quad 1 \leqq s \leqq k-1 .
$$

In light of Lemma 3.1 and the bounds (1.12c) for $\operatorname{div} \underset{\sim}{\xi},(1.12 \mathrm{~b})$ has been verified. This completes the derivation of the bounds given in (1.12).

In order to demonstrate the $L^{\infty}$ bound of (1.14), let us impose the additional constraint (1.13) on the triangulation $\mathscr{T}_{h}$. Then, for any element $\underline{v}$ in $V_{h}$, the inverse inequality

holds. Since, from (3.11),

$$
\|\underline{v}\|_{0, \infty} \leqq Q h^{-1}\|\underline{v}\|_{0}
$$

then

$$
\|\underline{\gamma}\|_{0} \leqq Q\|\underline{u}\|_{r+1} h^{r+1} \quad \text { for } 1 \leqq r \leqq k+1,
$$

$$
\begin{aligned}
\left\|\underline{u}-\underline{\sim}_{h}\right\|_{0, \infty} & \leqq\left\|\underline{u}-P_{h} \underset{u}{u}\right\|_{0, \infty}+\|\underline{\gamma}\|_{0, \infty} \\
& \leqq Q\left\{\|\underline{u}\|_{r, \infty}+\|\underline{u}\|_{r+1}\right\} h^{r}
\end{aligned}
$$

for $1 \leqq r \leqq k+1$, where any reasonable form of the Bramble-Hilbert lemma has been applied to obtain the $L^{\infty}$ approximation property of $P_{h}$.

The mixed method proposed here can also be analyzed using the abstract framework of Brezzi [2]. That method does not yield estimates so sharp as (1.12), but it does provide a quasioptimal estimate on $\underset{\approx}{H} \times \underset{\approx}{V}$. Such an estimate is useful for various purposes; e.g., for studying the effects of strong mesh 
refinement. We therefore briefly sketch the application of the theory of [2] to our method.

First, we incorporate the conditions (1.2c) and (1.3c) of Sect. 2 into the variational formulations by replacing $\underset{\approx}{H}$ in (1.2) and $\underset{\approx}{H}$ in (1.3) by $\underset{\approx}{\hat{H}}$ and $\underset{\tilde{W}}{\hat{H}}$, respectively, where the circumflex denotes the intersection with $\left\{\tilde{\tau} \in L_{\approx}^{2}(\Omega) \mid \int_{\Omega} \operatorname{tr}(\tau) d x=0\right\}$. The resulting variational problems are equivalent with the original continuous and discrete problems. That the first of Brezzi's conditions is verified follows immediately from Lemmas 3.1 and 3.2. It is easy to verify Brezzi's second condition by making use of the one-sided bounded invertibility of div: $\underset{\sim}{\hat{H}^{1}} \rightarrow \underset{V}{V}(1.7),(1.9 \mathrm{a})$, and the fact that $\underset{\approx}{\delta \in H_{h}}$. Consequently, we have the quasioptimal error estimate

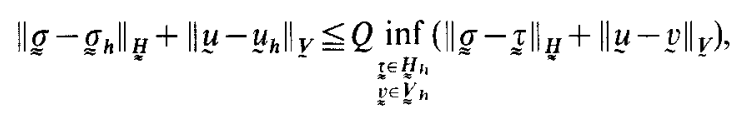

with $Q$ depending only on $\Omega, m, \mu_{0}, \mu_{1}$, and $k$. The infimum may be taken over $\underset{\approx}{H}$ rather than $\underset{\approx}{\hat{H}}$ since $\underset{\approx}{\sigma \in \underset{\sim}{H}}$ and so these infima are in fact equal.

\section{The Reference Composite Triangle}

Let $K$ be the triangle with vertices $a_{1}=(0,1), a_{2}=(0,0)$, and $a_{3}=(1,0)$. Let $a_{4}$ $=\left(\frac{1}{3}, \frac{1}{3}\right)$ be its centroid, and divide $K$ into the three triangles $T_{1}, T_{2}$, and $T_{3}$, where $T_{i}$ has as its vertices $a_{4}$ and $\left\{a_{1}, a_{2}, a_{3}\right\} \backslash\left\{a_{i}\right\}$. Let

$$
\underline{V}^{k-1}={\underset{P}{k-1}}_{k-1}(K)
$$

the vectors with components being polynomials of degree $k-1$ on $K$. Let

$$
\begin{aligned}
& M_{\approx}^{k}=\left\{\underset{\approx}{\left\{\in P_{k}\right.}\left(T_{i}\right), i=1,2,3: \underset{\approx n}{\tau n}\right. \text { is continuous across } \\
& \text { the interior edges } \left.T_{i} \cap T_{j}, i \neq j\right\} \text {; }
\end{aligned}
$$

here $\underline{n}$ denotes a normal to $T_{i} \cap T_{j}$. Let

$$
W^{k}=\left\{\underset{\approx}{\tau} \in M^{k}: \operatorname{div} \underset{\approx}{\tau} \in \underline{V}^{k-1}\right\} .
$$

We shall seek $\underset{\approx}{H^{k}}$, the restriction of $\underset{\approx}{H}$ to $K$, as a subspace of $W^{k}$. Note that the divergence of each of our elements is a vector polynomial on $K$, not just on each $T_{i}$. Let

$$
N_{j}^{m}=\left\{\varphi \in C^{j}(K):\left.\varphi\right|_{T_{i}} \in P_{m}\left(T_{i}\right), i=1,2,3\right\} .
$$

Since the gradient of any function $\varphi$ in $N_{1}^{k+2}$ has continuous tangential derivatives across $T_{i} \cap T_{j}, i \neq j$, it follows that $\underset{\approx}{\operatorname{airy}}(\varphi) \in \underset{\approx}{M^{k}}$. Also, if $\underset{\approx}{\tau \in H} \underset{\approx}{H}$ is such that $\operatorname{div} \tau=0$, then there exists $\varphi \in H^{2}(K)$ such that $\underset{\approx}{\tau}=\operatorname{airy}(\varphi)$; moreover, if $\tau \in N_{\approx-1}^{k}$, then $\varphi \in N_{1}^{k+2}$ and it is determined up to an additive linear function.

Note that, for $k \geqq 2$,

$$
\operatorname{dim} M_{\approx}^{k} \geqq \operatorname{dim} \underset{\approx-1}{N_{-1}^{k}}-3 \cdot 2(k+1)=\frac{9}{2} k^{2}+\frac{15}{2} k+3,
$$


which we shall use to prove the following sequence to be exact (i.e., that the image of each map is the kernel of the following map):

$$
0 \rightarrow P_{1} \stackrel{\subset}{\longrightarrow} N_{1}^{k+2}-\stackrel{\text { dirv }}{\longrightarrow} M_{\approx}^{k} \stackrel{\text { div }}{\longrightarrow} N_{-1}^{k-1} \rightarrow 0 .
$$

Now, $\operatorname{dim} P_{1}=3$ and $\operatorname{dim}{\underset{\sim}{N-1}}_{-1}^{k-1}=3 k^{2}+3 k$. It is known [3] that $\operatorname{dim} N_{1}^{k+2}=\frac{3}{2} k^{2}$ $+\frac{9}{2} k+6$. Thus,

$$
\begin{aligned}
& \operatorname{dim} \operatorname{div} \underset{\approx}{M^{k}} \geqq\left(\frac{9}{2} k^{2}+\frac{15}{2} k+3\right)-\left(\frac{3}{2} k^{2}+\frac{9}{2} k+6\right)+3 \\
&=3 k^{2}+3 k=\operatorname{dim}{\underset{\approx}{N-1} k, 1}^{N-1}
\end{aligned}
$$

so that,

$$
\operatorname{dim} M_{\approx}^{k}=\frac{9}{2} k^{2}+\frac{15}{2} k+3
$$

and the sequence is exact.

In particular, $\operatorname{div} M_{\approx}^{k}=N_{-1}^{k-1}$, so that

$$
\begin{aligned}
\operatorname{dim} \underset{\approx}{W^{k}} & =\operatorname{dim} \underset{\sim}{P_{k-1}}+\operatorname{dim} \mathscr{N}\left(M_{\approx}^{k} \stackrel{\operatorname{div}}{\longrightarrow} N_{-1}^{k-1}\right) \\
& =\left(k^{2}+k\right)+\operatorname{dim} N_{1}^{k+2}-3 \\
& =\frac{5}{2} k^{2}+\frac{11}{2} k+3 .
\end{aligned}
$$

Next, we shall define a map

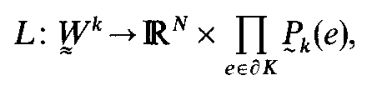

where $e$ varies over the external edges of $K$ and

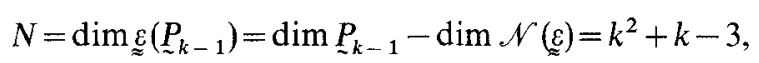

since the null space of $\xi$ is the set of vectors of the form $(a+c y, b-c x)$ for $(a, b, c) \in \mathbb{R}^{3}$. Let $L=\left(L_{1}, L_{2}\right)$ be defined by

$$
\begin{aligned}
& L_{1}(\tau)=\left\{\int_{K} \underset{\approx}{\tau}: \underset{\approx}{\rho} d x: \underset{\approx}{\rho} \in \operatorname{Basis}\left(\underset{\widetilde{z}}{\left.\left.\left(\underset{\sim}{P_{k-1}}\right)\right)\right\},}\right.\right. \\
& L_{2}(\tau)=\left\{\tau n_{e}: e \text { an edge of } \partial K\right\} .
\end{aligned}
$$

The map $L$ will define some (all if $k$ is two or three) of the degrees of freedom $\stackrel{H}{z}^{k}$, at least when $(4.2 \mathrm{~b})$ is interpreted to correspond to the specification of the moments

$$
\left\{\int_{e} \underset{\sim}{\tau n_{e}} \cdot \psi d s: \underset{\sim}{\psi P_{k}}(e), e \text { an edge of } \partial K\right\}
$$

cf. (1.10).

Lemma 4.1. The map $L: W^{k} \rightarrow \mathbb{R}^{N} \times \prod_{e \in \partial K} \underset{\sim}{P}(e)$ is onto.

Proof. It suffices to show that

$$
\operatorname{dim} \mathscr{N}(L)=\operatorname{dim} W^{k}-(N+6 k+6)=\frac{3}{2} k^{2}-\frac{3}{2} k .
$$


Let $\underset{\tau}{\tau} \in \mathcal{N}(L)$. Then, $\operatorname{div} \underset{\sim}{\tau \in P_{k-1}}$, and for any $\rho \in P_{k-1}$,

$$
\int_{K} \operatorname{div}_{\sim} \underset{\approx}{\tau} \cdot \rho d x=-\int_{K} \tau: \underset{\approx}{\tau}(\rho) d x+\int_{\partial K}(\tau n) \cdot \rho d s=0,
$$

so that $\operatorname{div}_{\sim} \tau=0$. Hence, there exists $\varphi \in N_{1}^{k+2}$, unique up to an additive linear function, such that $\tau=\underset{\approx}{\operatorname{airy}}(\varphi)$. Now,

$$
\underset{\approx}{\tau}=\underset{\approx}{\operatorname{airy}}(\varphi) \underline{n}=\frac{\partial}{\partial s} \operatorname{curl} \varphi=0
$$

on each edge of $\partial K$. Thus, curl $\varphi$ is constant on each edge; as $\varphi \in C^{1}(K), \operatorname{curl} \varphi$ is constant on $\partial K$. By choosing the exactly linear part of the arbitrary linear function properly, we can assume that $\frac{\partial \varphi}{\partial x}$ and $\frac{\partial \varphi}{\partial y}$ vanish on $\partial K$. Thus, $\varphi$ is constant on $\partial K$, and we can then impose the condition that $\varphi=0$ on $\partial K$; i.e.,

$$
\varphi \in\left\{\psi \in N_{1}^{k+1}: \psi=\frac{\partial \psi}{\partial n}=0 \text { on } \partial K\right\}=: \stackrel{\circ}{1}_{1}^{k+2} .
$$

The composite triangle elements $N_{1}^{k+2}$ are the generalized Clough-Tocher elements of Douglas, Dupont, Percell, and Scott $[3,9]$, and a useful set of degrees of freedom is known for $N_{i}^{k+2}$. The function and its gradient can be specified at each (external) vertex of $K$, and $k-1$ values of the function and $k$ values of its normal derivative can be given at points on the interior of each edge. These degrees of freedom can be completed to form a unisolvent set. Hence,

$$
\operatorname{dim} \stackrel{\circ}{1}_{1}^{k+2}=\operatorname{dim} N_{1}^{k+2}-3\{3+(k-1)+k\}=\frac{3}{2} k^{2}-\frac{3}{2} k .
$$

Since $\stackrel{N}{1}_{1}^{k+2}$ contains no nontrivial linear functions, the airy operator maps this space one-to-one onto $\mathscr{N}(L)$, and the lemma has been demonstrated.

We are now able to indicate the choice of $\underset{\approx}{H^{k}}$. We require that ${\underset{\approx}{H}}^{k}$ be a minimal subspace of $W^{k}$ such that

$$
\begin{aligned}
& L\left({\underset{\approx}{H^{k}}}^{k}\right)=R^{N} \times \prod_{e \in \partial K} P_{k}(e) \\
& \underset{\approx}{P}(K) \subset \underset{\approx}{H^{k}} .
\end{aligned}
$$

The dimension of ${\underset{\approx}{*}}^{k}$ can be determined as follows. Let $\underset{\approx}{\tau} \mathcal{N}(L) \cap \underset{\approx}{P_{k}}(K)$. Then, as above, $\tau=\operatorname{airy}(\varphi)$, where $\varphi \in P_{k+2}(K)$ and $\varphi=\partial \varphi / \partial n=0$ on $\partial K$. Thus, if the barycentric coordinates of $K$ are denoted by $\left(\lambda_{1}, \lambda_{2}, \lambda_{3}\right)$, the function $\lambda_{1}^{2} \lambda_{2}^{2} \lambda_{3}^{2}$ divides $\varphi$. Clearly, if $k=2$ or $3, \varphi$ must vanish, and $\left.L\right|_{p_{k}(K)}$ is one-to-one. In general,

so that

$$
\mathscr{N}\left(\left.L\right|_{P_{k}(K)}\right)=\underset{\approx}{\operatorname{airy}}\left(\lambda_{1}^{2} \lambda_{2}^{2} \lambda_{3}^{2} P_{k-4}(K)\right)
$$

$$
\operatorname{dim} \mathscr{N}\left(\left.L\right|_{P_{k}(K)}\right)=\frac{k^{2}}{2}-\frac{5}{2} k+3, \quad k \geqq 2 .
$$


Thus,

$$
\begin{aligned}
\operatorname{dim} \underset{\approx}{H^{k}} & =\operatorname{dim}\left(\mathbb{R}^{N} \times \prod_{\in \in \partial K} \underset{\sim}{P_{k}}(e)\right)+\operatorname{dim} \mathscr{N}\left(L_{P_{k}(K)}\right) \\
& =\frac{3}{2} k^{2}+\frac{9}{2} k+6 \\
& =\operatorname{dim} \underset{\approx}{P_{k}}(K)+3
\end{aligned}
$$

i.e., $H_{\Sigma}^{k}$ is the span of the tensor polynomials of degree $k$ on $K$ and exactly three additional functions, which must be composite, for each $k \geqq 2$. Moreover, the degrees of freedom given by (1.10) indeed form a unisolvent set for $H_{\approx}^{k}$.

It is clear that there is a certain degree of arbitrariness in the way $\underset{\approx k}{\underset{2}{P}}(K)$ is augmented to obtain $H^{k}$. We shall outline what seems to us to be a natural way to select three necessarily composite basis elements to complete a definition of ${\underset{\xi}{k}}^{k}$. We shall add a further requirement for $\underset{\tilde{*}}{H^{k}}$; however, the space will not be uniquely determined. Let us ask that $H^{k^{2}}$ be invariant under affine transformations of $K$ onto itself; i.e., if $F: K \rightarrow K, F(x)=B x+b$, is an affine map of $K$ onto itself, let [8]

$$
\left(F_{*} \tau\right)(x)=B \underset{\approx}{\tau}\left(F^{-1}(x)\right) B^{T}, \quad x \in K,
$$

define the associated covariant transformation of tensor-valued functions. Then the additional constraint takes the form

$$
F_{*}\left({\underset{\approx}{H}}^{k}\right)=\underset{\approx}{H^{k}}
$$

for all affine $F$ mapping $K$ onto itself. Since the restriction of $\underset{z}{\not} H_{h}$ to triangles other than the reference one will be defined from ${ }_{\tilde{z}}{ }^{k}$ using an affine map, this additional condition is necessary if the resulting space is to be independent of the choice of the affine map from the reference element.

Note that the degrees of freedom defined by $(1.10 \mathrm{a})$ can be replaced by the specification of the values of the normal vector $\tau n$ at the end points of each edge and at $k-1$ equally spaced interior points on each edge. Let us choose a tensor $\mathfrak{\tau}^{\prime} \in W^{k}$ such that, if $n_{3}=(-1,0)$ is the normal to the edge $a_{1} a_{2}$,

$$
\underline{\tau}^{\prime} \underline{n}_{3}=(0,1) \text { at }(0,0+)
$$

all other degrees of freedom of $\tau_{\approx}^{\prime}$ vanish.

Since $\mathfrak{z}^{\prime} n_{3} \cdot n_{1}=(0,1) \cdot(0,-1)=-1$ at $(0,0+)$ and $\tau^{\prime} n_{1} \cdot n_{3}=(0,0) \cdot(-1,0)=0$ at $(0+, 0), \widetilde{\tau^{\prime}}$ is discontinuous at $a_{2}$ and cannot belong to $\underset{z}{P_{k}}(K)$. The tensor $\tau^{\prime}$ is not uniquely determined, as will be discussed below.

Let $F^{0}$ indicate the affine map such that $F^{0}\left(\left(a_{1}, a_{2}, a_{3}\right)\right)=\left(a_{3}, a_{2}, a_{1}\right)$, and let $F^{1}$ be the one such that $F^{1}\left(\left(a_{1}, a_{2}, a_{3}\right)\right)=\left(a_{2}, a_{3}, a_{1}\right)$. Let

$$
\underset{\approx}{H^{k}}=\underset{\approx}{P}(K)+\operatorname{Span}\left[\tau_{\approx}^{\prime}, F_{*}^{1} \underline{\tau}^{\prime},\left(F_{*}^{1}\right)^{2} \tau^{\prime}\right]
$$

From a dimensional count it is clear that $L\left(P_{k}(K)\right)$ is equal to the 3codimensional subspace of $\mathbb{R}^{N} \times \prod_{e \in \partial K} P_{k}(e)$ determined by the condition of continuity of $\underset{\approx}{\tau} n_{i} \cdot \underline{n}_{j}$ at $a_{k}$ for $\{i, j, k\}=\{1,2,3\}$. It follows that the space defined in 
(4.6) does indeed satisfy (4.3) for any choice of $\tau^{\prime} \in W^{k}$ with the degrees of freedom specified in (4.5). The further condition (4.4) is satisfied if and only if

$$
F_{*}^{0}{\stackrel{\tau}{\tau} \in{\underset{\approx}{H}}^{k}}^{k}
$$

Now consider the tensor $\underset{\tilde{\tau}}{\tau^{*}}=\underset{\widetilde{\tau}}{\tau^{\prime}}+F_{*}^{0} \underset{\tilde{\tau}}{\tau^{\prime}}$. One easily verifies that ${\underset{\tau}{*}}^{*} \underline{n}_{i} \cdot \underline{\sim}_{j}$ is continuous at $a_{k}$ for $\{i, j, k\}=\{1,2,3\}$. Hence, there exists a unique polynomial tensor $\underset{z}{\tau} \in \underset{z}{P_{k}}(K)$ with the same degrees of freedom. This tensor can be calculated. For example, if $k=2$, then

$$
\tau_{0}=\left(\begin{array}{cc}
0 & -1+3(x+y)-2(x+y)^{2} \\
-1+3(x+y)-2(x+y)^{2} & 0
\end{array}\right) .
$$

Now, $\underset{\approx}{\tau} \in \underset{\approx}{P_{k}}(K) \subset \underset{\approx}{H^{k}}$ and the degrees of freedom (1.10) are unisolvent on $\underset{\tilde{\sigma}}{H^{k}}$, so (4.7) holds if and only if

$$
{\underset{\tau}{\tau}}^{\prime}+F_{*}^{0} \tau^{\prime}=\underset{\approx}{\tau}
$$

To summarize: the minimal dimensional subspaces of $W^{k}$ satisfying (4.3) and (4.4) are exactly those spaces (4.6) corresponding to $\underset{\approx}{\tau^{\prime}}$ with degrees of freedom (4.5) and satisfying (4.8).

Three questions thus naturally arise: first, does there exist such a ${\underset{\tau}{\tau} \in}_{\tilde{\tau}} \in W^{k}$; second, what is the dimension of the affine space of all such $\underset{\tilde{\tau}}{\tau^{\prime}}$; and, third, how can $\tau_{\tilde{\tau}}^{\prime}$ be constructed in a form suitable for practical computation. The answer to the first question is affirmative: if $\underset{\tau}{\tau} \in W^{k}$ is any element with the degrees of freedom specified in (4.5), then

$$
\stackrel{\tau}{\tau}^{\prime}=\left(\tau+\underset{\approx}{\tau}-F_{*}^{0} \tau\right) / 2
$$

has the same degrees of freedom and also satisfies (4.8). On the other hand, $\mathfrak{\tau}^{\prime}$ is not unique. It is determined up to the addition of any $\rho \in W^{k}$ which has all degrees of freedom vanishing and satisfies the homogeneous̃ equation.

$$
\underset{\approx}{\rho}+F_{*}^{0} \underset{\approx}{\rho}=0 \text {. }
$$

The space of all such $\underset{\approx}{\rho}$ is a nontrivial linear subspace of $\underset{\approx}{\operatorname{airy}}\left(\stackrel{\circ}{N}_{1}^{k+2}\right)$ with the nonboundary degrees of freedom chosen to ensure (4.9) as well as orthogonality to airy $\left(\lambda_{1}^{2} \lambda_{2}^{2} \lambda_{3}^{2} P_{k-4}(K)\right)$. In the case $k=2$ this space has dimension one; it is spanned by $\underset{\approx}{\rho}=\underset{\approx}{\operatorname{airy}}(\varphi)$ where $\varphi$ is the quartic Clough-Tocher element $[3,9]$ with boundary degrees of freedom zero, value zero at $a_{4}$, and gradient $(-1,1)$ at $a_{4}$. We do not know of a criterion for preferring one particular choice of $\tau^{\prime}$ over another.

To construct the general solution $\underset{\tilde{\tau}}{\tau^{\prime}}$, that is, to determine its coefficients with respect to a basis for $N_{-1}^{k}$, one can use standard computer linear algebra subroutines. In the case $k \stackrel{\approx}{=}$, a particular solution calculated in this way is given in Table $1\left(2 \underset{\tau}{\tau^{\prime}}\right.$ is presented as it has integer coefficients while $\underset{\tau}{\tau^{\prime}}$ does not). The coefficients of the function $\rho$ referred to in the above paragraph are displayed in Table $2 \underset{\approx}{(\rho / 162}$ is displayed for convenience). Thus, the space $\underset{\approx}{H^{2}}$ is 
given in (4.6), where $\tau^{\prime}$ is specified in Table 1 up to an additive multiple of the function specified in Table 2. The coefficients of the elements of a complete nodal basis can then be easily calculated.

Table 1. The coefficients of the basis element $2 \tau^{\prime}$

\begin{tabular}{llrrrrrr}
\hline Triangle & Component & 1 & $x$ & $y$ & $x^{2}$ & $x y$ & $y^{2}$ \\
\hline 1 & $(1,1)$ & 2 & 2 & -24 & -4 & 2 & 48 \\
& $(1,2)$ & 0 & 0 & -2 & 0 & 8 & -1 \\
& $(2,2)$ & 0 & 0 & 6 & 0 & -8 & -8 \\
2 & $(1,1)$ & 0 & -2 & 0 & 2 & 2 & 0 \\
& $(1,2)$ & -1 & 4 & 2 & -3 & -4 & -1 \\
3 & $(2,2)$ & 0 & 0 & 2 & 0 & -2 & -2 \\
& $(1,1)$ & 0 & -6 & 0 & 8 & 8 & 0 \\
& $(1,2)$ & -2 & 8 & 6 & -3 & -16 & -4 \\
& $(2,2)$ & -2 & 24 & -2 & -48 & -2 & 4 \\
\hline
\end{tabular}

Table 2. The coefficients of an element having vanishing degrees of freedom

\begin{tabular}{llrrrrrr}
\hline Triangle & Component & 1 & $x$ & $y$ & $x^{2}$ & $x y$ & $y^{2}$ \\
\hline 1 & $(1,1)$ & 0 & -1 & 3 & 1 & 2 & -10 \\
& $(1,2)$ & 0 & 0 & 1 & 0 & -2 & -1 \\
2 & $(2,2)$ & 0 & 0 & 0 & 0 & 0 & 1 \\
& $(1,1)$ & -1 & 1 & 3 & 0 & -2 & -2 \\
& $(1,2)$ & 0 & 1 & -1 & -1 & 0 & 1 \\
3 & $(2,2)$ & 1 & -3 & -1 & 2 & 2 & 0 \\
& $(1,1)$ & 0 & 0 & 0 & -1 & 0 & 0 \\
& $(1,2)$ & 0 & -1 & 0 & 1 & 2 & 0 \\
& $(2,2)$ & 0 & -3 & 1 & 10 & -2 & -1 \\
\hline
\end{tabular}

\section{The Finite Element Spaces}

Fix the index $k \geqq 2$, and consider again a triangulation $\mathscr{T}_{h}$ satisfying (1.8). With each $K \in \mathscr{T}_{h}$ we associate an affine invertible $\operatorname{map} F=F_{K}$ taking the vertices $(0,1),(0,0)$, and $(1,0)$ of the reference triangle onto the vertices of $K$, with $(0,0)$ being mapped onto a vertex lying in $\Omega$. Let $\hat{K}=F^{-1}(K)$. Thus, $\hat{K}$ is a curvilinear triangle with vertices $(0,1),(0,0)$, and $(1,0)$ and two edges along the coordinate axes and with at most one curved edge. Its shape depends on $K$. However, by (1.8d) there exists a positive constant $C$ depending only on $\theta$ such that $\hat{K}$ is star-shaped with respect to a disc of radius $C$. Through the addition of $(1 / 3,1 / 3)$ as vertex $\hat{K}$ is partitioned into three triangles, one possibly curvilinear, in the obvious way.

In the last section we constructed the space of piecewise polynomial tensors $H_{\approx}^{k}$ on the (straight-edged) referenced triangle. Let $\underset{\approx}{H}(\widehat{K})=\underset{\approx}{H}(k, \widehat{K})$ be the space 
of tensor functions on $\hat{K}$ which are polynomial on each of its three microtriangles and which coincide with an element $H^{k}$ on the intersection of $\hat{K}$ with the reference triangle. (Thus, if $K$ is a straight-edged, $\underset{\approx}{H}(\hat{K})=H_{\approx}^{k}$.)

We now define the restrictions of $V_{h}$ and $H_{h}$ to $K$ by

$$
\begin{aligned}
& \underset{V}{V}(K)={\underset{\sim}{k-1}}_{k}(K), \quad K \in \mathscr{T}_{h}, \\
& \underset{\approx}{H}(K)=\left\{F_{*} \underset{\approx}{\hat{\tau}}: \underset{\approx}{\hat{\tau} \in \underset{\approx}{H}(\hat{K})\} .}\right.
\end{aligned}
$$

Note that, when $K$ has a curved edge, the polynomials in $V(K)$ and the piecewise polynomials in $\underset{\sim}{H(K)}$ are exactly the same as would result for a straight-edged triangle with the same vertices. Finally, set

$$
\begin{aligned}
& V_{h}=V_{k}\left(k, \mathscr{T}_{k}\right)=\left\{v \in V:\left.\underline{v}\right|_{K} \in V(K), K \in \mathscr{T}_{h}\right\}, \\
& {\underset{\approx}{H}}_{h}=\underset{\approx h}{H_{h}}\left(k, \mathscr{T}_{h}\right)=\left\{\tau \in \underset{\approx}{H}:\left.\tau\right|_{K} \in \underset{\approx}{H}(K), K \in \mathscr{T}_{h}\right\} .
\end{aligned}
$$

Note that (1.5) holds as a result of (4.1), (5.1), and the properties of the transformation $F_{*}[8]$.

\section{The Projection $\Pi_{h}$}

It remains to construct the projection operator $\Pi_{h}$ and to verify the conditions (1.4), (1.9a), and (1.9c). The approximation property $(1.9 \mathrm{c})$ follows from a standard argument based on the Bramble-Hilbert lemma since the constant in that lemma depends only on the shape of the elements $K \in \mathscr{T}_{h}$ through the parameter $\theta$ of $(1.8 \mathrm{~d})$ [6].

To construct the operator $\Pi_{h}$ it suffices to construct for each $K \in \mathscr{T}_{h}$ an operator

$$
\Pi(K):{\underset{\approx}{H}}^{1}(K) \rightarrow \underset{\approx}{H}(K)
$$

with the following properties for $\underset{\approx}{\tau \in H^{1}}(K)$ :

$$
\begin{gathered}
\left(\tau-\Pi(K) \underset{\approx}{(\tau)} \underset{\sim}{n} \text { is orthogonal to } \underset{\sim}{P_{k}}(e) \text { on all edges } e\right. \text { of the } \\
\text { triangulation interior to } \Omega,
\end{gathered}
$$

For, then we can define $\Pi_{h} \tau$ for $\underset{\approx}{\tau \in H_{\approx}}(\Omega)$ by

$$
\left.\left(\Pi_{h} \tau\right)\right|_{K}=\Pi(K)\left(\left.\tau\right|_{K}\right), \quad K \in \mathscr{T}_{h} .
$$

By (6.1 a) and (5.1 b), $\Pi_{h \approx} \tau \in H_{h}$ (see [8]). The property (1.4) is an immediate consequence of $(6.1 \mathrm{~b})$, and $(1.9 \mathrm{a})$ follows directly from $(6.1 \mathrm{c})$.

The definition of $\Pi(K)$ will be different depending on whether $K$ has a curved edge or not. In either case we define $\hat{K}$ and $F: \hat{K} \rightarrow K$ as in Sect. 5 and define first $\Pi(\hat{K}): H_{\approx}^{1}(\hat{K}) \rightarrow H(\hat{K})$ by certain orthogonalities. Let $\underset{\approx}{\hat{\tau}} \in H^{1}(\hat{K})$ be arbitrary and set $\rho=\hat{\tau}-\Pi(\hat{K}) \hat{\tau}$. 
If $K$ is straight-edged we define $\Pi(\hat{K}) \tau$ by

$\underset{z}{\rho} \underline{n}$ is orthogonal to $\underset{\approx}{P}(e)$ on each of the edges of $\hat{K}$, $\underset{\approx}{\rho}$ is orthogonal to $\left.\underset{\approx}{\varepsilon\left(P_{k-1}\right.}(\hat{K})\right)+\underset{\approx}{\operatorname{airy}}\left(\lambda_{1}^{2} \lambda_{2}^{2} \lambda_{3}^{2} \mathbf{P}_{k-4}(\hat{K})\right)$ on $\hat{K}$.

We have seen in Sect. 4 that $\Pi(\hat{K})$ is well-defined. Note that for $\psi \in P_{k-1}(K)$,

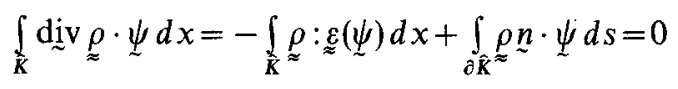

by $(6.2 \mathrm{a})$ and $(6.2 \mathrm{~b})$; i.e.,

$$
\operatorname{div}_{\sim} \underset{\sim}{\rho} \text { is orthogonal to } \underset{\sim}{P_{k-1}}(\hat{K}) .
$$

For $K \in \mathscr{T}_{h}$ with a curved edge we shall impose the condition (6.2a) only on the interior edges. This will allow us to impose (6.3) directly. The remaining orthogonalities will be against the space

$$
\underset{\approx}{Y(\hat{K})}=\{\underset{\approx \in}{\sigma}(\hat{K}): \underset{\approx}{g}=0 \text { on the straight edges of } \hat{K} \text { and } \operatorname{div} g=0\} .
$$

Note that the piecewise polynomials in $\underset{*}{Y}(\hat{K})$ do not depend on the element $K$. One easily sees that

$$
\operatorname{dim} \underset{\approx}{Y}(\hat{K})=\operatorname{dim} \underset{\approx}{H}(\hat{K})-2 \operatorname{dim}{\underset{\sim}{x}}_{k}(\mathbb{R})-\operatorname{dim}{\underset{\sim}{P}-1}_{k}=\frac{k^{2}}{2}-\frac{k}{2}+2 .
$$

For $\underset{\approx}{\hat{\tau} \in \underset{\tilde{*}}{H}}{ }^{1}(\hat{K})$ we define $\Pi(\hat{K}) \underset{\approx}{\tau} \in \underset{\approx}{H}(\hat{K})$ by the following orthogonalities of $\underset{\approx}{\rho}=\underset{\approx}{\widehat{\tau}}$ $-\Pi(\widehat{K}) \tau$ :

$$
\underset{\sim}{\rho n} \text { is orthogonal to } \underset{\sim}{P}(e) \text { on the two straight edges } e \text { of } \hat{K},
$$

$$
\begin{gathered}
\operatorname{div} \underset{\approx}{\rho} \text { is orthogonal to } \underset{\sim}{P_{k-1}}(\hat{K}), \\
\underset{\approx}{\rho} \text { is orthogonal to } \underset{\approx}{Y}(\hat{K}) .
\end{gathered}
$$

These conditions uniquely determine $\Pi(\hat{K}) \tau$. Indeed we have imposed $\operatorname{dim} \underset{\approx}{H}(\hat{K})$ linear conditions and if $\underset{\approx}{\rho} \in \underset{\approx}{H}(\hat{K})$ satisfies $(6.4)$, then $\underset{\approx}{\rho} \in(\hat{K})$ by $(6.4 \mathrm{a}, \mathrm{b})$, so that $\rho=0$ by $(6.4 \mathrm{c})$.

We now define $\Pi(K)$ via the affine transformation $F: \hat{K} \rightarrow K$ :

$$
\Pi(K) \underset{\approx}{\tau}=F_{*}(\Pi(\hat{K}) \hat{\tau}), \quad \tau=F_{*} \hat{\tau} \in H_{\approx}^{1}(K) .
$$

Then (6.1 a) follows from (6.2a) and (6.4a), and (6.1 b) follows from (6.3) and $(6.4 \mathrm{~b})$. Finally $(6.1 \mathrm{c})$ is established by a straightforward argument using the Bramble-Hilbert lemma.

\section{The Composite Quadrilateral}

Let $Q$ be a convex quadrilateral with vertices $\left(a_{1}, a_{2}, a_{3}, a_{4}\right)$, and triangulate it into the four triangles $T_{1}, \ldots, T_{4}$ by means of the diagonals. Let 


$$
\begin{aligned}
V^{k-1}(Q)= & \underset{*-1}{P_{k-1}}(Q), \\
M^{k}(Q)= & \left\{\underset{\approx}{\sim} \in P_{k}\left(T_{i}\right), i=1, \ldots, 4: \underset{\approx n}{\tau n}\right. \text { is continuous across } \\
& \text { the interior edges } \left.T_{i} \cap T_{j}, i \neq j\right\}, \\
W^{k}(Q)= & \left\{\underset{\approx}{\tau \in M^{k}}: \operatorname{div}_{\approx} \tau \in V^{k-1}\right\}, \\
N_{j}^{m}= & \left\{\varphi \in C^{j}(Q):\left.\varphi\right|_{T_{i}} \in P_{m}\left(T_{i}\right), i=1, \ldots, 4\right\} .
\end{aligned}
$$

The object again is to define the restriction $\underset{\approx}{H}(k, Q)$ of $\underset{\approx}{H}$ to $Q$ as a subspace of $W^{k}$. The argument parallels that of Sect. 4 .

First, it is clear that

In fact,

$$
\operatorname{dim} M_{\approx}^{k} \geqq \operatorname{dim} \underset{\approx}{N_{-1}^{k}}-8(k+1)=6 k^{2}+10 k+4
$$

$$
\operatorname{dim} M_{\approx}^{k}=6 k^{2}+10 k+5
$$

as there is a redundancy in the $8(k+1)$ constraints used to insure that $\tau n$ be continuous across interior edges. To see this, it suffices to consider a quadrilateral as shown in the figure below.

Let $\underset{\tau}{\tau}=\left(\tau_{i j}^{k}\right)$ on $T_{k}$. Then, the continuity of $\underset{\approx}{\tau} n$ leads to the relations (where $\underline{n}$ $=\left(n_{1}, n_{2}\right), n_{1} \neq 0$, is the normal to $\left.T_{1} \cap T_{2}\right)$

$$
\begin{aligned}
& \tau_{12}^{1}=\tau_{12}^{4}, \quad \tau_{22}^{1}=\tau_{22}^{4} \quad \text { on } T_{1} \cap T_{4}, \\
& \tau_{12}^{2}=\tau_{12}^{3}, \quad \tau_{22}^{2}=\tau_{22}^{3} \quad \text { on } T_{2} \cap T_{3} \text {, } \\
& n_{1} \tau_{11}^{1}+n_{2} \tau_{12}^{1}=n_{1} \tau_{11}^{2}+n_{2} \tau_{12}^{2} \text {, } \\
& n_{1} \tau_{12}^{1}+n_{2} \tau_{22}^{1}=n_{1} \tau_{12}^{2}+n_{2} \tau_{22}^{2} \text { on } T_{1} \cap T_{2} \text {, } \\
& n_{1} \tau_{11}^{3}+n_{2} \tau_{12}^{3}=n_{1} \tau_{11}^{4}+n_{2} \tau_{12}^{4} \text {, } \\
& n_{1} \tau_{12}^{3}+n_{2} \tau_{22}^{3}=n_{1} \tau_{12}^{4}+n_{2} \tau_{22}^{4} \quad \text { on } T_{3} \cap T_{4} \text {. }
\end{aligned}
$$

If $n_{2}=0$, then (7.2a)-(7.2c) imply that $\tau_{12}^{1}=\tau_{12}^{2}=\tau_{12}^{3}=\tau_{12}^{4}$, at the origin, which implies that the second equation of (7.2d) holds at the origin. In fact, it is easily seen that this latter implication is valid also when $n_{2} \neq 0$. Thus, (7.2) represents at most $8(k+1)-1$ independent linear relations among the coefficients of the $\tau_{i j}^{k}$, and so $\operatorname{dim} \underset{\approx}{M}>6 k^{2}+10 k+5$; that equality holds will result from showing that the sequence

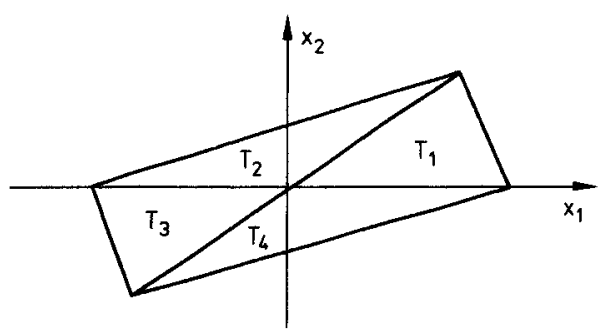

Fig. 1 


$$
0 \rightarrow P_{1} \stackrel{\subset}{\longrightarrow} N_{1}^{k+2}-\stackrel{\text { airry }}{\longrightarrow} \underset{\approx}{M^{k} \stackrel{\text { div }}{\longrightarrow}} \underset{\sim}{N_{-1}^{k-1} \rightarrow 0}
$$

is exact. Since [3] $\operatorname{dim} N_{1}^{k+2}=2 k^{2}+6 k+8$ and $\operatorname{dim} N_{-1}^{k-1}=4 k^{2}+4 k$, the exactness follows as before and requires (7.1) to hold.

Next,

$$
\operatorname{dim} \underset{\approx}{W^{k}}=\operatorname{dim} \underset{\sim}{\underset{k-1}{P}}+\operatorname{dim} \mathscr{N}\left({\underset{\approx}{M}}^{k} \stackrel{\text { dịv }}{\longrightarrow} \underset{\sim-1}{N_{-1}^{k-1}}\right)=3 k^{2}+7 k+5 .
$$

Let us define degrees of freedom by the map

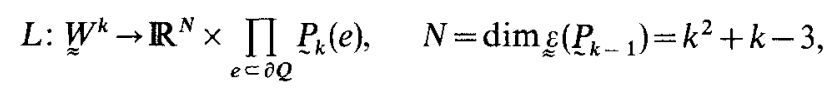

for $k \geqq 2$, where

$$
\begin{aligned}
& L_{1}(\tau)=\left\{\int_{Q} \underset{\approx}{\tau}: \underset{\approx}{\rho} d x: \underset{\approx}{\rho} \in \operatorname{Basis}\left(\varepsilon_{\mathcal{E}}\left(\underline{P}_{k-1}\right)\right)\right\}, \\
& L_{2}(\tau)=\left\{\underset{\tau}{\tau} \underline{e}_{e}: e \text { an edge of } \partial Q\right\} .
\end{aligned}
$$

That $L$ is onto can be demonstrated using the proof of Lemma 4.1 with the generalized Clough-Tocher element being replaced by the generalized Fraeijs de Veubeke-Sander element [3].

Here we want to choose $H(k, Q)$ to be a minimal subspace of $W^{k}$ such that $L(\underset{\approx}{H})=L\left(\underset{\approx}{W^{k}}\right)$ and $\underset{\approx}{H} \supset \underset{\approx}{P_{k}}(\tilde{Q})$. Its dimension can be computed in a similar fashion as for the composite triangle case. It is easy to see that, if $\underset{\approx}{\tau} \operatorname{ker}(L) \cap \underset{\approx}{P_{k}}(Q)$, then $\underset{\approx}{\tau}=\underset{\approx}{\operatorname{airy}}(\varphi), \varphi=\lambda_{1}^{2} \lambda_{2}^{2} \lambda_{3}^{2} \lambda_{4}^{2} \psi$, for some $\psi \in P_{k-6}(Q)$. Thus, (7.3a) and (7.3b) provide a complete set of degrees of freedom for $k<6$; it is necessary to supplement these relation by

$$
L_{3}(\tau)=\left\{\int_{Q} \underset{\approx}{\tau}: \underset{\approx}{\rho} d x: \underset{\approx}{\rho} \in \operatorname{Basis}\left(\underset{\approx}{\operatorname{airy}} \lambda_{1}^{2} \lambda_{2}^{2} \lambda_{3}^{2} \lambda_{4}^{2} P_{k-6}(Q)\right\}\right.
$$

for $k \geqq 6$. In particular,

Hence,

$$
\operatorname{dim} \operatorname{ker}\left(\left.L\right|_{P_{k}(Q)}\right)=\frac{1}{2}(k-4)(k-5), \quad k \geqq 4 .
$$

$$
\operatorname{dim}{\underset{\approx}{H}}^{k}= \begin{cases}27=\operatorname{dim} \underset{\approx}{P_{2}}(Q)+9, & k=2, \\ 41=\operatorname{dim} \underset{\approx}{P_{3}}(Q)+11, & k=3, \\ \frac{3}{2} k^{2}+\frac{9}{2} k+15=\operatorname{dim} \underset{\approx}{P_{k}}(Q)+12, & k \geqq 4 .\end{cases}
$$

As for the triangular element, such a space $\underset{\approx}{H}(k, Q)$ can be computed. Again it is not uniquely determined, and this nonuniqueness is greater than before.

From (7.3) it is clear how to define the projection operator $\Pi(Q)$ satisfying the properties analogous to (6.1). It is then obvious how to define the space $V_{h}$ and $H_{h}$ and the projection operator $\Pi_{h}$, and it is easy to verify the hypotheses of Sect. 1. The practicality of this quadrilateral element is unclear to the authors. 


\section{References}

1. Babuška, I., Aziz, A.K.: Survey lectures on the mathematical foundations of the finite element method. In: The mathematical foundations of the finite element method with applications to partial differential equations (A.K. Aziz, ed.). New York: Academic Press 1972

2. Brezzi, F.: On the existence, uniqueness and approximation of saddle point problems arising from Lagrangian multipliers. R.A.I.R.O. Anal. Numér. 2, 129-151 (1974)

3. Douglas, J., Dupont, T., Percell, P., Scott, R.: A family of $C^{1}$ finite elements with optimal approximation properties for various Galerkin methods for 2 nd and 4th order problems. R.A.I.R.O. Anal. Numér. 13, 227-255 (1979)

4. Douglas, J., Roberts, J.E.: Mixed finite elements methods for second order elliptic problem. Matemática Applicade e Computacional 1, 91-103 (1982)

5. Douglas, J., Roberts, J.E.: Global estimates for mixed methods for second order elliptic equations. (To appear in Math. Comput.)

6. Dupont, T., Scott, R.: Polynomial approximation of functions in Sobolev spaces. Math. Comput. 34, 441-463 (1980)

7. Falk, R.S., Osborn, J.E.: Error estimates for mixed methods. R.A.I.R.O., Anal. Numér. 14, 309324 (1980)

8. Johnson, C., Mercier, B.: Some equilibrium finite element methods for two-dimensional elasticity problems. Numer. Math. 30, 103-116 (1978)

9. Percell, P.: On cubic and quartic Clough-Tocher finite elements. SIAM J. Numer. Anal. 13, 100-103 (1976)

10. Raviart, P.A., Thomas, J.M.: A mixed finite element method for 2 nd order elliptic problems. Mathematical aspects of the finite element method. Lecture Notes in Mathematics 606. BerlinHeidelberg-New York: Springer 1977

11. Scholz, R.: $L_{\infty}$-convergence of saddle-point approximation for second order problems. R.A.I.R.O. Anal. Numér. 11, 209-216 (1977)

12. Scholz, R.: A remark on the rate of convergence for a mixed finite element method for second order problems. Numer. Funct. Anal. and Optimiz. 4, 269-277 (1981/82)

13. Scholz, R.: Optimal $L_{\infty}$-estimates for a mixed finite element method for second order elliptic and parabolic problems. (To appear in Calcolo)

14. Temam, R.: Navier-stokes equations. Amsterdam: North Holland 1977

15. Thomas, J.M.: Sur l'analyse numérique des méthodes d'éléments finis mixtes et hybrides. Thèse, Paris 1977

16. Vogelius, M.: An analysis of the p-version of the finite element method for nearly incompressible materials. Uniformly valid, optimal order error estimates. Numer. Math. 41, 39-53 (1983)

Received August 9, 1983 / March 14, 1984 